\title{
RADIO ASTROMETRY OF THE CLOSE ACTIVE BINARY HR 5110
}

\author{
E. AbBuhl ${ }^{1}$, R. L. Mutel ${ }^{1}$, C. LyNCH $^{1}$, AND M. GÜedel ${ }^{2}$ \\ ${ }^{1}$ Department of Physics and Astronomy, University of Iowa, Van Allen Hall, Iowa City, Iowa 52242, USA \\ 2 Department of Astronomy, University of Vienna, Vienna, Austria \\ Received 2015 March 26; accepted 2015 August 15; published 2015 September 17
}

\begin{abstract}
The close active binary HR 5110 was observed at six epochs over 26 days using a global very long baseline interferometry array at $15.4 \mathrm{GHz}$. We used phase referencing to determine the position of the radio centroid at each epoch with an uncertainty significantly smaller than the component separation. After correcting for proper motion and parallax, we find that the centroid locations of all six epochs have barycenter separations consistent with an emission source located on the KIV secondary, and not in an interaction region between the stars or on the F primary. We used a homogeneous power-law gyrosynchrotron emission model to reproduce the observed flux densities and fractional circular polarization. The resulting ranges of mean magnetic field strength and relativistic electron densities are of the order of $10 \mathrm{G}$ and $10^{5} \mathrm{~cm}^{-3}$, respectively, in the source region.
\end{abstract}

Key words: astrometry - binaries: close - stars: chromospheres - stars: magnetic field

\section{INTRODUCTION}

Late-type stars in short-period binary systems often exhibit signposts of enhanced magnetic activity. These include persistent photospheric spots, ultraviolet chromospheric emission, X-ray emission from hot coronae, and non-thermal radio emission (e.g., Guinan \& Giménez 1993). The conventional interpretation is that tidal effects enforce spin-orbit coupling, and the consequent rapid rotation drives a robust magnetic dynamo on one or both components of the system (e.g., Schrijver \& Zwaan 1991).

Close active binaries are categorized by whether one of both companions fill their respective Roche lobes. The eponymous RS CVn systems have both components detached and no mass transfer (Hall et al. 1978), while Algol systems are semidetached with one component (typically the cooler, more evolved star) filling its Roche lobe, leading to episodic mass transfer. Finally, contact binaries have both components within each other's Roche lobes and continuously transfer mass. Curiously, radio luminosities do not differ greatly among these three classes (Umana et al. 1998), while the mean X-ray luminosity of Algol-class systems is 3-4 times lower than RS CVn's (Singh et al. 1996). This suggests that dynamical processes, such as mass transfer, and accretion disks may be decoupled from emission processes related to enhanced magnetic fields.

An important open question for these systems is whether the magnetically driven enhanced emission arises primarily from a single active component or from a magnetic interaction region between the stars. Very long baseline radio interferometry (VLBI) provides a powerful method to address this question, since for nearby $(d \lesssim 100 \mathrm{pc})$ systems it can probe the structure of the radio coronae at spatial scales smaller than the binary orbit. A number of close active binaries have been studied using VLBI arrays for several decades (e.g., Clark et al. 1976; Lestrade et al. 1984, 1988; Mutel et al. 1984, 1985; Massi et al. 1988; Trigilio et al. 1993; Lebach et al. 1999; Ransom et al. 2003). However, locating the radio structure within the binary system requires high-precision astrometric techniques such as rapidly switching between the target star and one or more extragalactic phase reference sources with precisely determined positions. Multi-epoch observations over several years are needed in order to simultaneously solve for the astrometric parameters (parallax, proper motion, orbital motion) with sub-mas accuracy.

The first measurements of this type were made by Lestrade et al. (1993), who showed that the radio centroid of Algol's inner binary moved with the KIV secondary. Subsequent astrometric VLBI studies of Algol (Peterson et al. 2010, 2011) confirmed the K-star association. They also showed that during flares, the radio structure consisted of a large coronal loop approximately one stellar diameter in height, with a base straddling the sub-giant and oriented toward the B star. Other recent astrometric VLBI studies of close active binaries include UX Arietis (Peterson et al. 2011) and IM Peg (Ransom et al. 2012). In all three cases, the radio emission is associated with the more evolved star, a K subgiant or giant.

In this paper, we report on an astrometric VLBI study of a fourth close active binary. HR 5110 (BH CVn, HD118216) is a short-period (2.61 day) binary composed of an F2 IV primary and a K2 IV secondary. The orbit is circular and is oriented nearly face-on toward the observer (Eker \& Doherty 1987). This orientation is well suited to investigate whether the radio emission is associated with a single component or with an interaction region. It also provides a convenient test of active region association with polar spots (Huenemoerder et al. 2009), assuming that the magnetic and rotational axes are parallel and normal to the orbital plane, and hence oriented toward the observer.

Although no spots have been directly detected on HR 5110 , Little-Marenin et al. (1986) found that the IR spectrum can be best fit by assuming a persistent spot on the K secondary with a filling factor of $\sim 0.25$. It has been categorized both as an RS CVn system (Hall et al. 1978) and an Algol system (LittleMarenin et al. 1986), although UV observations have found no direct evidence of an accretion flow or disk. However, LittleMarenin et al. (1986) argue that the secondary must fill its Roche lobe based on the spectral classification and its close binary separation.

There have been several estimates of the size and location of the active region in HR 5110. Previous VLBI studies of the radio corona (Mutel et al. 1985; Ransom et al. 2003) found a "core-halo" structure, with a compact core size of the order of 
the K-star radius and a more extended halo somewhat larger than the orbital separation. This is similar to the results of Umana et al. (1993), who measured the radio spectrum of HR 5110 between 1.4 and $15 \mathrm{GHz}$ during several epochs of moderate activity. They found that the spectral energy distribution could be fit with a two component gyrosynchrotron model consisting of a higher density core with a size of about one-half the K-star radius and a lower density extended component approximately the size of the binary system.

Observations at other wavelengths provide additional constraints on the active region's size and location. LittleMarenin et al. (1986) found that UV emission lines are best fit with the $\mathrm{K}$ secondary's chromosphere and transition region, hence locating UV flares on the K star. Mullan et al. (2006) modeled UV flare observations with a compact active loop whose length was smaller than 0.42 stellar radii. However, Graffagnino et al. (1995) argued that the active region may be much larger. They fit ROSAT X-ray data from a large outburst lasting more than three days. They found that a very large emission region, on the scale of the binary separation, is required to fit the observed light curve. They suggest that the flare may have been driven by magnetic reconnection in the interbinary region between the stars. Assuming the radio, UV, and X-ray emission regions share a common active region site, an open question exists. Where in the binary system does this region lie?

We observed HR 5110 at multiple epochs using an intercontinental phase-referenced VLBI array. The observations were made at a frequency of $15 \mathrm{GHz}$, which provided twice the angular resolution of any previous VLBI study of this system. The primary goal of the observations was to determine the location of the radio emission within the binary system. We also modeled the observed emission properties (flux density, circular polarization, angular size) using a power-law gyrosynchrotron emission model to derive constraints on plasma parameters in the source region.

\section{OBSERVATIONS AND DATA ANALYSIS}

HR 5110 was observed at six epochs of 10 hours each with a global VLBI array consisting of the $1025 \mathrm{~m}$ telescopes of the Very Long Baseline Array (VLBA) ${ }^{3}$ and the $100 \mathrm{~m}$ Effelsberg telescope near Bonn, Germany. We scheduled observations with a cadence that allowed sampling at approximately evenly spaced orbital phases. The receivers sampled $64 \times 2 \mathrm{MHz}$ channels using 2-bit sampling centered at $15.4 \mathrm{GHz}$ in dual polarization mode. The synthesized beam was 0.4 mas in its smallest dimension which is approximately one-fifth the binary's major axis (Table 1).

All epochs were calibrated and imaged using the NRAO Astronomical Image Processing Software package (AIPS, Greisen 2003). Standard VLBI amplitude and delay-rate corrections were applied, followed by the transfer of complex gain corrections from the primary calibrator $(\mathrm{J} 1308+3546)$ to the target (HR 5110) and secondary phase calibrator (J1310 +3220 ; Diamond 1995). We assumed a flux density of $0.6 \mathrm{Jy}$ for $\mathrm{J} 1308+3546$. After calibration, we imaged all sources using Fourier inversion and numerical deconvolution (AIPS task IMAGR using the Clark CLEAN algorithm). We used the

\footnotetext{
3 The National Radio Astronomy Observatory is a facility of the National Science Foundation operated under cooperative agreement by Associated Universities, Inc.
}

Table 1

HR 5110 Properties And Orbital Elements

\begin{tabular}{|c|c|c|c|}
\hline Parameter & Symbol & Value & References $^{\mathrm{a}}$ \\
\hline \multicolumn{4}{|c|}{ Fixed values } \\
\hline Parallax & $\Pi$ & $22.21 \mathrm{mas}$ & 1 \\
\hline Spectral type & $\cdots$ & $\mathrm{F} 2 \mathrm{IV}+\mathrm{K} 2 \mathrm{IV}$ & 2 \\
\hline Primary mass & $m_{A}$ & $1.5 M_{\odot}$ & 2 \\
\hline Secondary mass & $m_{B}$ & $0.8 M_{\odot}$ & 2 \\
\hline Primary radius & $r_{A}$ & $2.6 R_{\odot}(0.27 \mathrm{mas})$ & 2 \\
\hline Secondary radius & $r_{B}$ & $3.4 R_{\odot}(0.35 \mathrm{mas})$ & 2 \\
\hline $\begin{array}{l}\text { Reference epoch } \\
\text { [phase } 0.0]^{\mathrm{b}}\end{array}$ & $T$ & 2445766.655 & 2 \\
\hline Eccentricity & $e$ & 0.00 & 2 \\
\hline Inclination $^{\mathrm{e}}$ & $i$ & $171: 1$ & 2 \\
\hline Semimajor axes & $a_{1}, a_{2}$ & $\begin{array}{l}0.017 \mathrm{AU} \text { (0.38 mas), } \\
0.032 \mathrm{AU} \text { (0.71 mas) }\end{array}$ & 2 \\
\hline $\begin{array}{l}\text { Component } \\
\text { separation }\end{array}$ & $a_{1}+a_{2}$ & 0.049 AU (1.09 mas) & 2 \\
\hline Period & $P$ & 2.613214 day & 3 \\
\hline \multicolumn{4}{|c|}{ Derived values } \\
\hline $\begin{array}{l}\text { Longitude of } \\
\text { ascending node }\end{array}$ & $\Omega$ & $89^{\circ} \pm 10^{\circ}$ & \\
\hline R.A. proper motion & $\mu_{\alpha} \cos \delta$ & $85.62 \pm 0.07{\text { mas } \mathrm{yr}^{-1}}^{-1}$ & \\
\hline decl. proper motion & $\mu_{\delta}$ & $-9.68 \pm 0.07{\text { mas } \mathrm{yr}^{-1}}^{-1}$ & \\
\hline Fiducial $^{\mathrm{d}}$ R.A. & $\alpha_{0}$ & $13: 34: 47.75949 \pm 0.00008$ & \\
\hline Fiducial $^{\mathrm{d}}$ Decl. & $\delta_{0}$ & $37: 10: 56.7605 \pm 0.0006$ & \\
\hline
\end{tabular}

Notes.

a References: (1) Lestrade et al. (1999), (2) Eker \& Doherty (1987), (3) Mayor \& Mazeh (1987).

${ }^{\mathrm{b}}$ Secondary is in conjunction (in front; Eker \& Doherty 1987).

c Assumes radio centroid is centered on $\mathrm{K}$ subgiant (see text).

${ }^{\mathrm{d}}$ Center of mass position at fiducial epoch $(\mathrm{JD}=2449028.000)$.

e Inclination from Eker \& Doherty (1987), but corrected for CW rotation (c.f Section 3).

nodding phase-referencing scheme (Lestrade et al. 1990), cycling at three-minute intervals between $\mathrm{J} 1308+3546$ and HR 5110. We observed $\mathrm{J} 1310+3220$ once every 15 minutes to check the stability of the phase-referencing scheme.

The nodding phase-referencing technique requires accurate interpolation of phases from calibrator scans to target scans. This proved to be problematic, both because of the relatively large angular separation ${ }^{4}$ between phase calibrator and HR $5110\left(5^{\circ} .5\right)$ and also because all epochs were scheduled during summer months, which often results in large shorttimescale phase fluctuations caused from wet-component tropospheric variations (Pradel et al. 2006). Although we were able to successfully transfer phase solutions from calibrator to target scans, it required careful inspection and flagging of excessive phase jumps (AIPS task SNFLG). We flagged all time-baseline visibilities for which the phase difference between adjacent $30 \mathrm{~s}$ calibrator phase solutions exceeded $60^{\circ}$, indicating a possible phase wrap ambiguity over the three-

\footnotetext{
4 Previous phase-referenced VLBI observations of HR 5110 (Lestrade et al. 1999; Ransom et al. 2003) used several angularly closer phase calibrator sources, but at lower frequencies $(5,8 \mathrm{GHz})$. To check their suitability at $15 \mathrm{GHz}$, in 2012 April we made "snapshot" VLBA observations of several angularly nearby compact sources. We found that the previously used sources were heavily resolved at $15 \mathrm{GHz}$ and therefore unusable, and that the closest suitable compact source was $\mathrm{J} 1308+3546$.
} 
Table 2

J2000 Heliocentric Positions

\begin{tabular}{|c|c|c|c|c|c|}
\hline $\mathrm{JD}$ & Year & R.A. (13:34:+) & decl. $(37: 10:+)$ & Phase References $^{\mathrm{a}}$ & References $^{\mathrm{b}}$ \\
\hline \multicolumn{6}{|c|}{ Archival and Previously Published } \\
\hline 2449028.000 & 1993.108 & $47.75947 \pm 0.00003$ & $56.7601 \pm 0.00070$ & OP326 & 1 \\
\hline 2451276.833 & 1999.266 & $47.80374 \pm 0.00020$ & $56.7003 \pm 0.00200$ & $\mathrm{~J} 1340+3754$ & 2 \\
\hline 2451631.831 & 2000.239 & $47.81060 \pm 0.00004$ & $56.6915 \pm 0.00060$ & $\mathrm{~J} 1317+3425$ & 3 \\
\hline 2454560.812 & 2008.258 & $47.86794 \pm 0.00003$ & $56.6129 \pm 0.00030$ & $\mathrm{~J} 1340+3754$ & 4 \\
\hline \multicolumn{6}{|c|}{ This Paper } \\
\hline 2456108.458 & 2012.495 & $47.89844 \pm 0.00002$ & $56.5721 \pm 0.00034$ & $\mathrm{~J} 1308+3546$ & 5 \\
\hline 2456118.438 & 2012.523 & $47.89852 \pm 0.00003$ & $56.5722 \pm 0.00017$ & $\mathrm{~J} 1308+3546$ & 5 \\
\hline 2456119.427 & 2012.525 & $47.89865 \pm 0.00001$ & $56.5725 \pm 0.00017$ & $\mathrm{~J} 1308+3546$ & 5 \\
\hline 2456122.417 & 2012.534 & $47.89867 \pm 0.00001$ & $56.5726 \pm 0.00017$ & $\mathrm{~J} 1308+3546$ & 5 \\
\hline 2456133.385 & 2012.564 & $47.89883 \pm 0.00001$ & $56.5728 \pm 0.00017$ & $\mathrm{~J} 1308+3546$ & 5 \\
\hline
\end{tabular}

Notes.

${ }^{\text {a }}$ Positions in reference to the calibrators: OP326 (R.A. $=13: 17: 36.49418$ decl. $\left.=34: 25: 15.9326\right)$, J1340+3754 (R.A. $=13: 40: 22.9518$ decl. $=37: 54: 43.835$ ), J1317 +3425 (R.A. $=13: 17: 36.4942$ decl. $=34: 25: 15.933)$, J1308+3546 (R.A. $=13: 08: 23.70911$ decl. $=35: 46: 37.164)$.

b References: 1. Lestrade et al. (1999), 2. VLBA archive code BG087, 3. VLBA archive code BB120, 4. VLBA archive S9644, 5. This paper.

minute nodding cycle. This resulted in more than half the visibilities in each $10 \mathrm{hr}$ observing epoch being flagged.

In spite of this significant data loss, the positional accuracy of the radio centroid positions was only modestly degraded. To establish the positional accuracy at each epoch, we measured variations in the measured position of the secondary phase calibrator $(\mathrm{J} 1310+3220$, angular separation $3: 3$ from $\mathrm{J} 1308$ +3546) compared with its ICRF position. At all epochs, except for epoch A (2012.495), the measured position was within \pm 0.1 mas of its ICRF position. This is consistent with the extrapolated uncertainty estimate of Pradel et al. (2006), who studied the astrometric accuracy of VLBA phase-referenced observations as a function of source-calibrator angular separation and declination for a range of tropospheric conditions.

The expected astrometric accuracy of phase referencing can be estimated by linearly extrapolating the rms position uncertainty trend given in Figure 2 of Pradel et al. (2006). For a source at declination $\delta=+25^{\circ}$, and source-phase reference angular separation $\Delta \theta$ degrees, at an observing frequency of $15 \mathrm{GHz}$ and with a significant wet tropospheric component, this can be written as

$$
\sigma(\Delta \theta) \sim 30 \mu \text { as } \times \Delta \theta
$$

where $\sigma$ is the positional uncertainty in micro-arcseconds for VLBA observations. Hence, the expected total $\mathrm{rms}$ positional uncertainty at $3: 3$ separation (primary-secondary calibrator) is 0.1 mas, in excellent agreement with our observations, and 0.17 mas at 5.5 separation (primary-target separation). We have used this estimate for the formal position uncertainties at epochs B-F. For epoch A, the peak-to-peak position jitter of the secondary calibrator was 0.2 mas, possibly resulting from an excessively wet troposphere at several telescopes. Scaling this uncertainty to $5: 5$ separation resulted in an estimated uncertainty of 0.33 mas for the position of HR 5110 at Epoch A.

The radio centroid could move by up to 0.7 mas over the course of each 10-hour observing epoch if the source were located at the KIV secondary. This position shift would significantly smear out the source brightness in maps made using the entire 10-hr observation. The motion can be corrected by adjusting the map phase center (using AIPS task CLCOR) with a model of the source location within the orbit versus time. This is problematic since the source position is not known a priori! In our case, this correction was unnecessary, since the flagging described above resulted in using only $\sim 3 \mathrm{hr}$ contiguous unflaggged time intervals at each epoch. These were typically near the end of each 10-hr observation. Because the source motion on this timescale is less than a resolving beam, there was no significant smearing effect.

In addition to the VLBI observations in this paper, we also calibrated and imaged five unpublished phase-referenced observations of HR 5110 from the public VLBA archive. Although these observations lack the spatial resolution and temporal sampling cadence to determine the position of the radio source within the binary orbit, the measured positions, along with the published position of Lestrade et al. (1999), allowed us to make a more precise determination of the proper motion of HR 5110.

\section{RESULTS}

We determined new proper motions for HR 5110 (Table 1) by combining our six-epoch position determinations with the previously published position of Lestrade et al. (1999) and positions that we determined from archival VLBA observations, as listed in Table 2. Our calculated right ascension proper motion is in good agreement with the Lestrade et al. (1999) value, while the declination proper motion is three standard deviations from their value.

Radio contour maps of HR 5110 at all six epochs are shown in Figure 1. Previous VLBI observations of HR 5110 found a core-halo source morphology at $5 \mathrm{GHz}$ (Mutel et al. 1985) and $8 \mathrm{GHz}$ (Ransom et al. 2003). The $15 \mathrm{GHz}$ maps are somewhat amorphous and do not manifest a clear core-halo structure even if the long baselines to the more sensitive Effelsberg telescope 

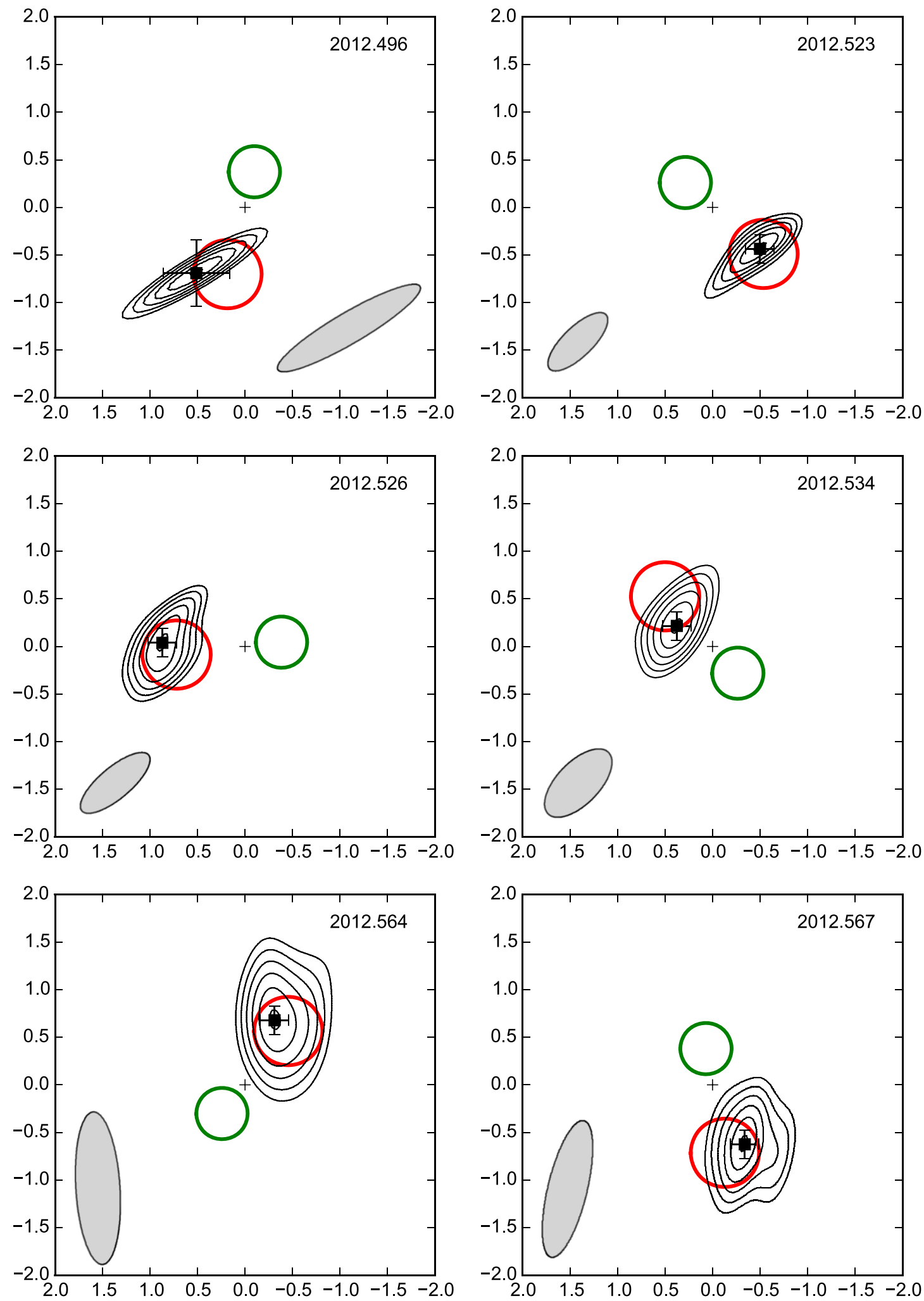

Figure 1. Radio contour maps of HR 5110 at $15 \mathrm{GHz}$ overlaid on the binary system in the center of mass frame of reference. The contour intervals are $50 \%$, $60 \%$, $70 \%, 80 \%$, and $90 \%$ of the maximum radio intensity at each epoch. The axes' label ticks are in mas relative to the binary center of mass. The superposed star positions (green circle $=$ FIV primary, red circle $=$ KIV secondary) are consistent with a least-squares minimization solution for a fixed location of the radio centroids (at all six epochs) in the corotating frame of the binary system (see Section 3). Since the resulting center of mass radial displacement was close to the K-star displacement, we identify the radio source location with the $\mathrm{K}$ secondary. The gray ellipse is the restoring beam at each epoch.

are excluded. However, we can characterize the spatial scale sizes and fluxes by fitting the images with a one component Gaussian. Fluxes vary significantly and are likely underestimates of the true flux due to the rapidly varying wet troposphere introducing significant phase errors (Martí-Vidal et al. 2010). All epochs were fit using a single component 
Table 3

Observed Fluxes and Gaussian Model Parameters

\begin{tabular}{|c|c|c|c|c|c|c|}
\hline Epoch & Code & $\Phi^{\mathrm{a}}$ & Stokes I (mJy) & Stokes $V / I$ & Diameter $(\mathrm{mas})^{\mathrm{b}}$ & $\log \left(T_{B}\right)$ \\
\hline 2012.523 & B & 0.37 & $7.7 \pm 0.8$ & $<0.08$ & $0.76 \pm 0.20$ & $7.8 \pm 0.1$ \\
\hline 2012.525 & $\mathrm{C}$ & 0.73 & $76.5 \pm 5.0$ & $+0.02 \pm 0.01$ & $0.86 \pm 0.05$ & $8.7 \pm 0.1$ \\
\hline 2012.564 & $\mathrm{E}$ & 0.11 & $12.2 \pm 0.9$ & $+0.18 \pm 0.01$ & $0.94 \pm 0.20$ & $7.9 \pm 0.1$ \\
\hline 2012.566 & $\mathrm{~F}$ & 0.47 & $10.3 \pm 1.6$ & $<0.12$ & $1.08 \pm 0.20$ & $7.7 \pm 0.1$ \\
\hline
\end{tabular}

Notes.

${ }^{a}$ Orbital phase computed using the period in Table 1 and adopting phase 0 at secondary conjunction (Eker \& Doherty 1987).

${ }^{\mathrm{b}}$ Sizes are the FWHM geometric means of elliptical single component Gaussian fits.

Gaussian and the resulting Gaussian model parameters are given in Table 3.

We used our measured proper motions, along with the parallax determined by Lestrade et al. (1999), to convert the observed radio centroids to displacements relative to the binary center of mass. These displacements were used to solve for the corotating location of the radio source with respect to the binary's center of mass. To do this, we assumed that the radio emission at all epochs is located at a fixed point in the corotating frame. We solved for this location by finding the point in the corotating frame that minimizes the sum of the squared distances (weighted by position uncertainty) from the center of mass to the radio centroids. Figure 2 shows the $\chi^{2}$ minimization surface as a function of the radial displacement from the center of mass and an azimuthal angle measured north through east in the sky plane. Table 4 lists the positional offset at each epoch between the measured radio centroid and the best-fit fixed point in the corotating frame.

The best-fit radial displacement is nearly the same as the semimajor axis of the K2 IV secondary, rather than the F2 IV primary or an interaction region midway between the stars. Since the binary's orientation on the sky is not known, the radio centroid azimuthal angles cannot be directly compared with the location of the component stars. However, given the close agreement between the model's radial displacement and the $\mathrm{K}$ star's semimajor axis, we make the plausible assumption that the radio centroids are located on or near the $\mathrm{K}$ star rather than a random co-moving location on the $\mathrm{K}$ star's orbit. Figure 3 shows the radio centroids and star positions in comoving sky plane coordinates, while Figure 4 is in the corotating frame, assuming the radio centroid-K star identification described above.

The identification of the radio centroid with the KIV secondary allows us to determine new values for two orbital elements of the binary system. First, since the observed radio centroid motion in the center of mass system is clockwise on the sky (Figure 3, black arrow on secondary orbit), the inclination angle is in the second quadrant (Heintz 1978). Hence, $i=171^{\circ} .1$ rather than 8.9 as given by Eker \& Doherty (1987). Second, the longitude of the ascending node (labeled $\Omega$ in Figure 3), which was previously unknown, was determined by using the epoch of secondary conjunction (Eker \& Doherty 1987, phase 0) to determine the interpolated radio position at phase 0.75 , i.e., the time of transit through the ascending node. We find $\Omega=89^{\circ} \pm 10^{\circ}$ where the uncertainty corresponds to the scatter in corotating radio centroid positions (Figure 4) translated to orbital phase.

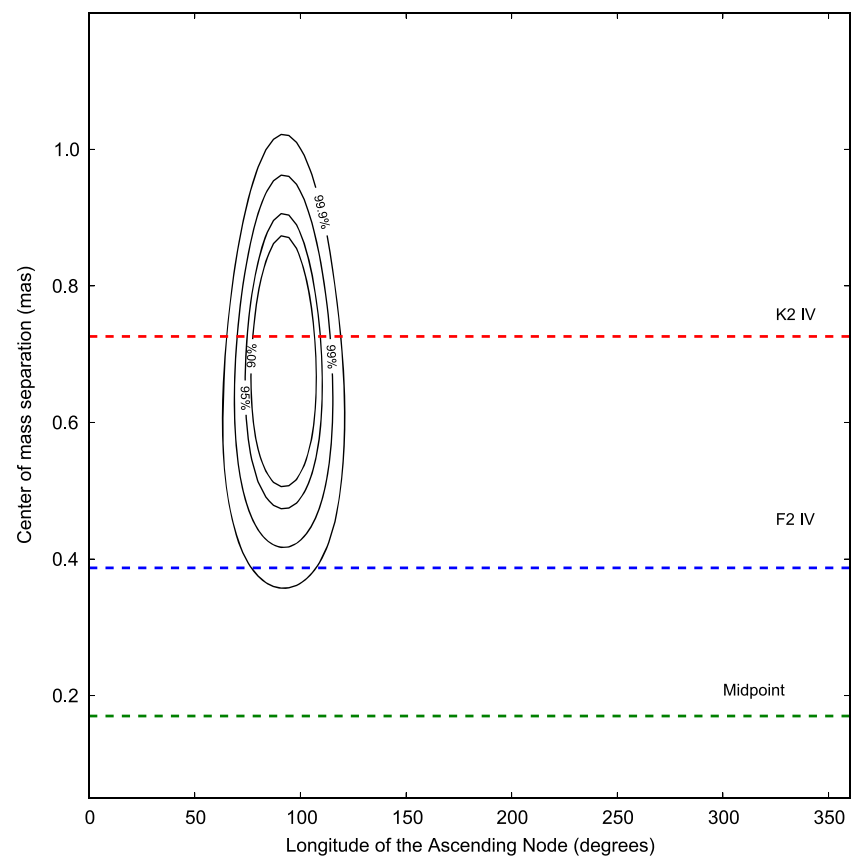

Figure 2. Confidence intervals of best-fit solution (center of mass displacement, ascending node angle) for stationary location of radio emission in the corotating frame. The dotted lines indicate the radial offsets from the center of mass for the K2 IV star (red), the F2 IV star (blue), and the midpoint between the two (green). Contours represent confidence intervals centered on the best-fit position $\left(r=0.70\right.$ mas, $\left.\Omega=89^{\circ}\right)$.

Table 4

Offsets From Best-fit Model Position

\begin{tabular}{lrr}
\hline \hline Epoch & $\Delta \alpha(\sigma)^{\mathrm{a}}$ & \multicolumn{1}{c}{$\Delta \delta(\sigma)^{\mathrm{a}}$} \\
\hline 1993.108 & $0.0(0.0)$ & $-0.9(1.3)$ \\
1999.250 & $1.1(0.6)$ & $-1.0(0.5)$ \\
1999.266 & $1.4(0.7)$ & $0.1(0.1)$ \\
2000.239 & $0.7(1.4)$ & $0.8(1.4)$ \\
2008.258 & $-0.3(1.0)$ & $-0.7(2.5)$ \\
2008.264 & $-0.5(1.7)$ & $0.3(1.1)$ \\
2012.495 & $0.6(3.9)$ & $-0.1(0.6)$ \\
2012.523 & $-0.2(0.6)$ & $0.1(0.3)$ \\
2012.525 & $0.2(1.5)$ & $0.0(0.0)$ \\
2012.534 & $-0.1(0.4)$ & $-0.4(2.6)$ \\
2012.564 & $0.0(0.1)$ & $0.1(0.8)$ \\
2012.566 & $0.0(0.2)$ & $0.0(0.1)$ \\
\hline
\end{tabular}

Note.

${ }^{\text {a }}$ Observed-model in mas (std. dev.). 


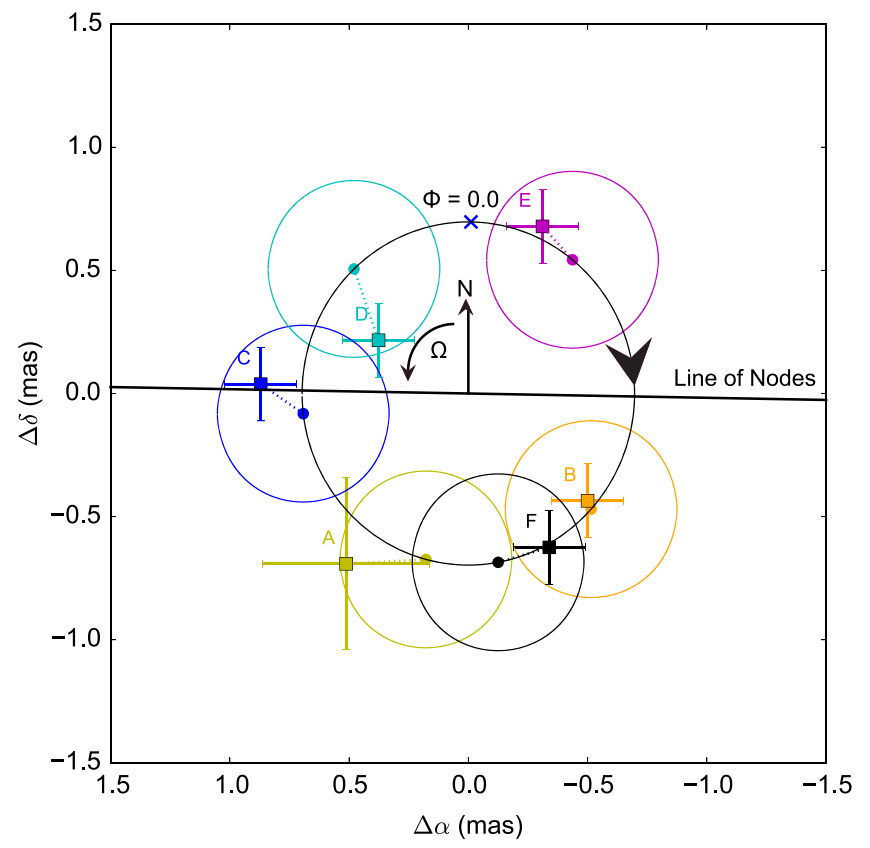

Figure 3. HR 5110 radio centroid locations at six epochs in the comoving center of mass system. Each epoch is color coded: A (yellow), B (orange), C (blue), D (cyan), E (magenta), and F (black). The large black circle is the orbit of the cooler KIV secondary star. Smaller colored circles show the secondary's size and location at the time of the corresponding observation. The dotted lines indicate the distance between the radio centroid and the KIV center at each epoch.

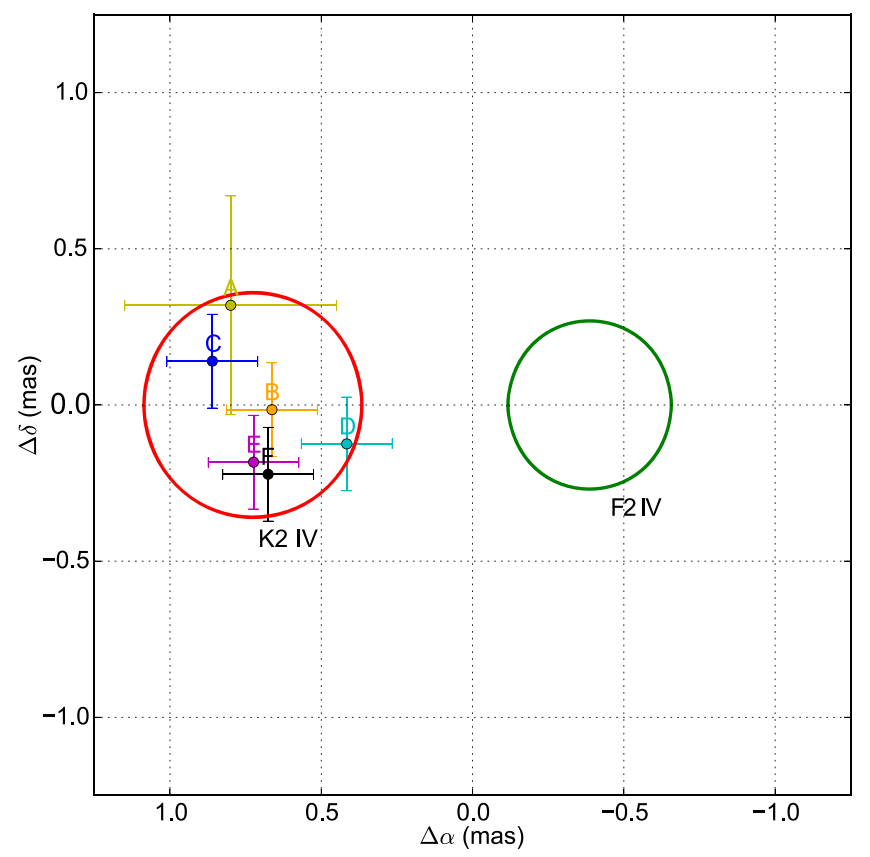

Figure 4. Radio centroid positions overlaid on the binary system in the corotating frame. The orientation of the stars is based on the close association of the best-fit radial offset with the K2 IV semimajor axis. Each epoch is color coded: A (yellow), B (orange), C (blue), D (cyan), E (magenta), and F (black). The two circles are the K2 IV star (red) and the F2 IV star (green).

\section{DISCUSSION}

\subsection{Active Region Location}

The principal astrometric result of this study is that the radio centroids of HR 5110 are located within one stellar radius of the corotating KIV secondary center at all epochs. The identification of the radio emission with the secondary component in close binaries has been seen previously in several other such systems, i.e., Algol (Lestrade et al. 1993; Peterson et al. 2010), UX Ari (Peterson et al. 2011), and HR 8703 (Ransom et al. 2012). Similarly, Little-Marenin et al. (1986) found that UV emission lines associated with an active chromosphere and transition region are located on the KIV secondary. By contrast, Graffagnino et al. (1995) modeled a very large X-ray flare from HR 5110 and found that the source size was comparable with the binary separation. They suggested that the interaction region between the stars could be the origin of the emission, e.g., the interbinary magnetic region posited for the binary CZ CVn (Strassmeier et al. 2011). In addition, during an extreme radio flare, Algol's emission was clearly displaced toward the hotter component (Peterson et al. 2010).

This apparent disagreement concerning the active region location poses an interesting conundrum. Are these systems driven by magnetic activity on the cooler star or between the stars? It is possible that the size and location of the active region depends on the intensity and duration of the flare. For all our VLBI epochs, the observed radio flux density of HR 5110 was significantly less than the highest recorded flux outburst ( $S>400$ mJy; Feldman \& Viner 1980). However, the large HR 5110 X-ray event and the large radio loop observed on Algol represent extraordinarily large outbursts. It is possible that moderate flares are located on the cooler secondary but that emission regions responsible for the largest flares can extend into, or arise from an interbinary region.

Previously, the radio emission was thought to be tied to a magnetically active polar spot (Elias et al. 1995), but the VLBI determined radio positions are not tightly grouped on a scale smaller than a stellar radius. Polar spots in binary systems are thought to be long lived, up to several years (Strassmeier 2009). By contrast the scatter of radio positions seen in Figure 4 occurs over a time period only slightly more than a month. In HR 5110 the orbital plane is inclined nearly perpendicular to the line of sight. If the magnetic pole of the cooler star is roughly aligned with its rotation axis then a putative polar spot would be nearly stationary from the observer's position. The observed scatter in the HR 5110 radio centroids is inconsistent with emission from a stationary location associated with a polar spot. This result for HR 5110 is very similar to HR 8703 (Ransom et al. 2012) where the radio centroids at multiple, well-separated epochs are scattered over the entire stellar disk.

\subsection{Physical Properties of Emission Region: Gyrosynchrotron Model}

To characterize the plasma conditions responsible for the observed radio emission, we constructed a simplified coronal model consisting of constant density, mildly relativistic powerlaw electrons in a uniform magnetic field emitting gyrosynchrotron emission. The emitting volume is a cube whose size is the average observed VLBI angular size $\left(0.6 \mathrm{mas}=5.7 R_{\odot}\right)$. This is clearly an oversimplified model, but allows for order-ofmagnitude estimates of the electron density and magnetic field strength without making unsubstantiated assumptions concerning the magnetic field topology or energetic electron population parameters.

The model solves for the emergent Stokes $I$ and $V$ flux density at the distance to HR 5110 (45 pc.). We used the 

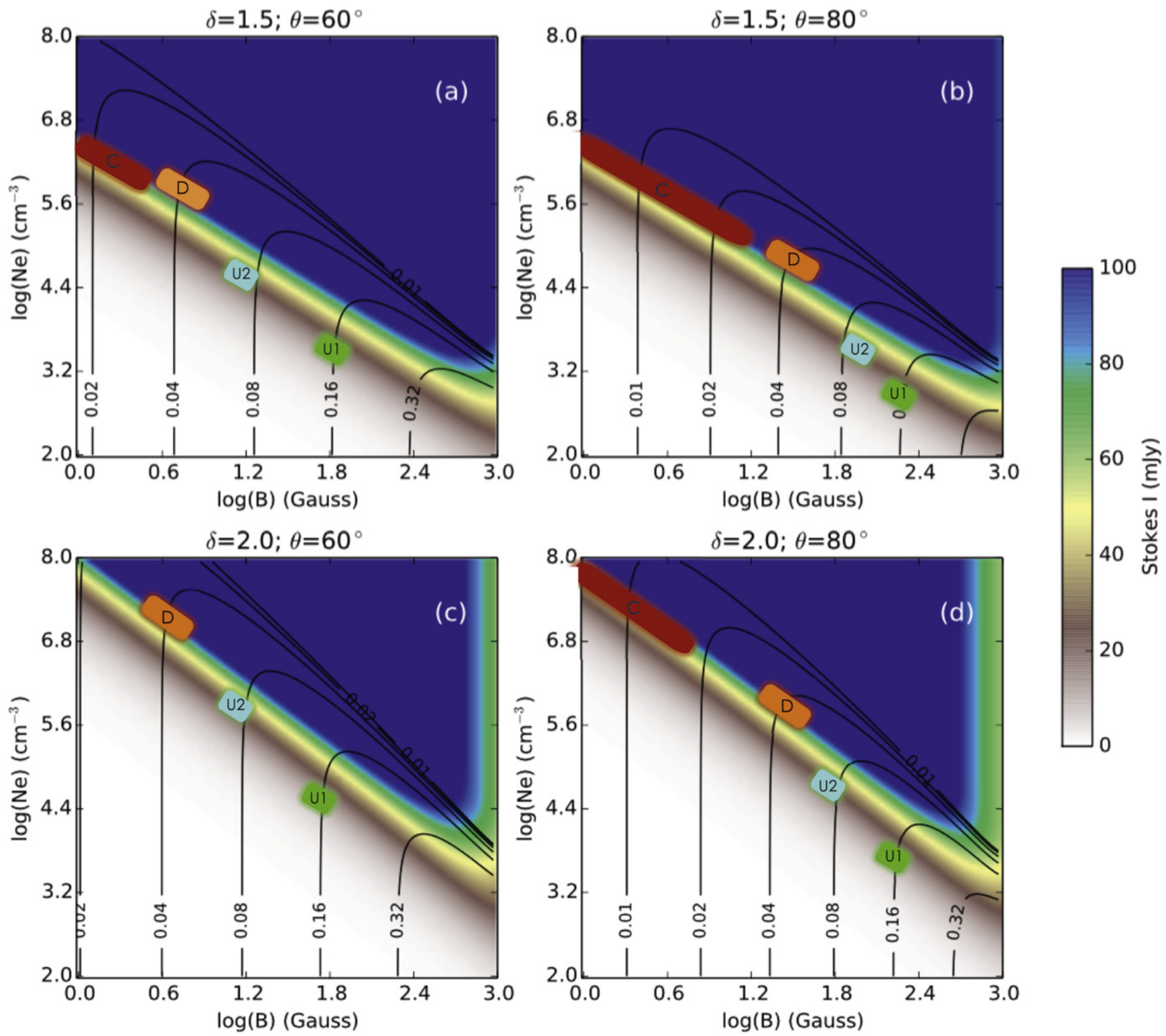

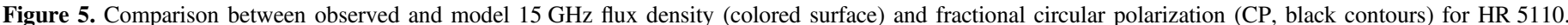

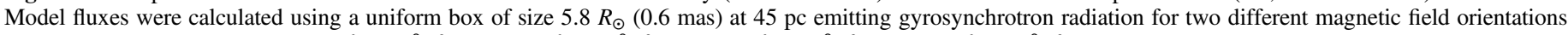

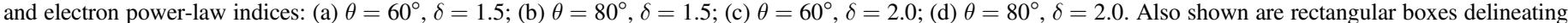

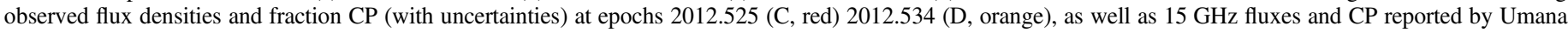
et al. (1993) at epochs 1989.134 (U1, green) and 1989.178 (U2, cyan).

expressions of Robinson \& Melrose (1984) for the emission and absorption coefficients of gyrosynchrotron emission from mildly relativistic electrons with a power-law electron energy distribution given by

$$
N_{e}(E)=K E^{-\delta},
$$

where $K=N_{0}(\delta-1) E_{0}{ }^{\delta-1}$ and is $\delta$ the energy index, and with a low-energy cutoff $E_{0}=10 \mathrm{keV}$. The emergent flux density was calculated by integrating the radiative transfer equation, summing the contributions along uniformly spaced lines of sight through the cube. We varied the strength and orientation of the magnetic field as well as the density and energy index of the relativistic electron population to best fit the measured $15 \mathrm{GHz}$ integrated flux density and fractional circular polarization at each epoch.
Figure 5 shows a surface plot of Stokes $I$ flux density as a function of electron density and magnetic field strength for four pairs of energy index and magnetic field orientation angle $\theta$ measured with respect to the observer's line of sight. Multifrequency observations of HR 5110 were obtained by Umana et al. (1993) and White et al. (1990). Both reported relatively flat spectral indices $(\alpha \leqslant-0.6)$ at $15 \mathrm{GHz}$. For a synchrotron process, the spectral index is simply related to the power-law electron energy index $\delta=1-2 \alpha \leqslant 2.2$, so we chose representative values 1.5 and 2.0. The magnetic field orientation is unconstrained by prior observations, but values smaller then $60^{\circ}$ resulted in model circular polarization fractions that exceeded our observed values, so we show only larger angles.

For a given Stokes $I$ flux density the solution is highly degenerate, with constant flux density values occurring along contours of nearly constant slope in $\log \left(N_{e}\right)-\log (B)$ 
coordinates. These lines have a slope that depends on primarily on the energy index, and can be approximated by

$$
\frac{\log \left(N_{e}\right)}{\log (B)} \sim-0.8 \delta \text {. }
$$

However, a measurement of circular polarization (CP) can break this degeneracy, as shown by the black contours, which are labeled by fractional CP. At epochs $\mathrm{C}$ and $\mathrm{D}$, the signal-tonoise ratio was sufficient to measure significant non-zero circularly polarized emission (Table 3), so we used these epochs to constrain the model. Observed flux densities and fractional circular polarization are shown as overlaid rectangles (red: epoch C, orange: epoch D), where the widths represent the uncertainty in each value. For comparison, we also show $15 \mathrm{GHz}$ flux densities and CP reported by Umana et al. (1993) at epochs 1989.134 (green, label U1) and 1989.178 (cyan, label U2).

Comparing the model with observed fluxes and $\mathrm{CP}$, we find that although there is still a significant parameter degeneracy, the present observations favor electron densities in the range $n_{e} \sim 10^{5} \mathrm{~cm}^{-3}$ and magnetic field strengths $B \sim 10$ Gauss, but with a large allowable range (approximately $\pm 1 \mathrm{dex}$ ). By comparison, the Umana et al. (1993) observations favor somewhat lower electron densities and higher magnetic field strengths. Since the source is highly variable, these differences may reflect different physical conditions in the emission region at different epochs.

\section{SUMMARY}

We observed the close active binary HR 5110 with a global VLBI array at $15.4 \mathrm{GHz}$ at six epochs distributed uniformly over the binary's orbit phase. The primary goal of these observations was to determine the location of the radio source within the binary system. To do this, we used a phasereferencing nodding scheme, rapidly switching from the target star to an angularly nearby extragalactic radio source.

After correcting for parallax and proper motion, we determined the locations of the radio centroids within the corotating binary system with sub-millarcsecond accuracy. The positions were scattered around a fixed location (within measurement uncertainty) in the corotating frame of the binary. The radial offsets from the center of mass were nearly the same as the semimajor axis of the KIV secondary, strongly suggesting the KIV secondary as the source of the radio emission. This identification of the radio source with the cooler, more evolved star has been previous reported for several other close binary systems, including Algol (Lestrade et al. 1999; Peterson et al. 2010), UX Ari (Peterson et al. 2011), and IM Peg (Ransom et al. 2012).

The clockwise radio centroid motion indicates an orbital inclination angle of $171^{\circ} .1$ rather than 8.9 as given by Eker $\&$ Doherty (1987). Additionally, the longitude of the ascending node $\left(\Omega=89^{\circ}\right)$ was determined using the identification of the radio centroids with the KIV secondary and the previously determined time of secondary conjunction (Eker \& Doherty 1987).

We used the observed properties of the radio emission (flux density, angular size, circular polarization) to constrain plasma parameters in the source region. The model used a simplified geometry consisting of power-law relativistic electrons in a uniform magnetic field radiating gyrosynchrotron emission. The derived values of magnetic field strength and relativistic electron density are degenerate, but the degeneracy is broken when there is a measured fractional circular polarization. For two such epochs, we find a mean magnetic field strength of the order of 10 Gauss and electron densities that vary from $10^{4}$ to $10^{6} \mathrm{~cm}^{-3}$ depending on the electron power-law index and magnetic field orientation.

\section{REFERENCES}

Clark, T. A., Hutton, L. K., Ma, C., et al. 1976, ApJL, 206, L107

Diamond, P. J. 1995, in ASP Conf. Ser. 82, Very Long Baseline Interferometry and the VLBA, ed. J. A. Zensus, P. J. Diamond \& P. J. Napier (San Francisco, CA: ASP), 227

Eker, Z., \& Doherty, L. R. 1987, MNRAS, 228, 869

Elias, N. M., Quirrenbach, A., Witzel, A., et al. 1995, ApJ, 439, 983

Feldman, P. A., \& Viner, M. R. 1980, BAAS, 12, 508

Graffagnino, V. G., Wonnacott, D., \& Schaeidt, S. 1995, MNRAS, 275, 129

Greisen, E. W. 2003, in Astrophysics and Space Science Library 285, Information Handling in Astronomy-Historical Vistas, ed. A. Heck (Dordrecht: Kluwer Academic Publishers), 109

Guinan, E. F., \& Giménez, A. 1993, in Astrophysics and Space Science Library 177, The Realm of Interacting Binary Stars, ed. J. Sahade, G. E. McCluskey, Jr. \& Y. Kondo (Dordrecht: Kluwer Academic Publishers), 51

Hall, D. S., Henry, G. W., Vaucher, C. A., et al. 1978, IBVS, 1459, 1

Heintz, W. D. 1978, Double Stars (Dordrecht: D. Reidel Publishing Co.)

Huenemoerder, D. P., Osten, R. A., Kesich, A., Testa, P., \& Schulz, N. 2009, in AIP Conf. Ser. 1094, 15th Cambridge Workshop on Cool Stars, Stellar Systems, and the Sun, ed. E. Stempels (Melville, NY: AIP), 656

Lebach, D. E., Ratner, M. I., Shapiro, I. I., et al. 1999, ApJL, 517, L43

Lestrade, J. F., Mutel, R. L., Phillips, R. B., et al. 1984, ApJL, 282, L23

Lestrade, J.-F., Mutel, R. L., Preston, R. A., \& Phillips, R. B. 1988, ApJ, 328,232

Lestrade, J.-F., Phillips, R. B., Hodges, M. W., \& Preston, R. A. 1993, ApJ, 410,808

Lestrade, J. F., Preston, R. A., Jones, D. L., et al. 1999, A\&A, 344, 1014

Lestrade, J. F., Rogers, A. E. E., Whitney, A. R., et al. 1990, AJ, 99, 1663

Little-Marenin, I. R., Linsky, J. L., \& Simon, T. 1986, in Cool Stars, Stellar Systems and the Sun, Vol. 254, ed. M. Zeilik \& D. M. Gibson (Berlin: Springer), 247

Martí-Vidal, I., Ros, E., Pérez-Torres, M. A., et al. 2010, A\&A, 515, A53

Massi, M., Felli, M., Pallavicini, R., et al. 1988, A\&A, 197, 200

Mayor, M., \& Mazeh, T. 1987, A\&A, 171, 157

Mullan, D. J., Mathioudakis, M., Bloomfield, D. S., \& Christian, D. J. 2006, ApJS, 164, 173

Mutel, R. L., Doiron, D. J., Phillips, R. B., \& Lestrade, J. F. 1984, ApJ, 278,220

Mutel, R. L., Lestrade, J. F., Preston, R. A., \& Phillips, R. B. 1985, ApJ, 289, 262

Peterson, W. M., Mutel, R. L., Güdel, M., \& Goss, W. M. 2010, Natur, 463, 207

Peterson, W. M., Mutel, R. L., Lestrade, J.-F., Güdel, M., \& Goss, W. M. 2011, ApJ, 737, 104

Pradel, N., Charlot, P., \& Lestrade, J. F. 2006, A\&A, 452, 1099

Ransom, R. R., Bartel, N., Bietenholz, M. F., et al. 2003, ApJ, 587, 390

Ransom, R. R., Bartel, N., Bietenholz, M. F., et al. 2012, ApJS, 201, 6

Robinson, P. A., \& Melrose, D. 1984, AuJPh, 37, 675

Schrijver, C. J., \& Zwaan, C. 1991, A\&A, 251, 183

Singh, K. P., Drake, S. A., \& White, N. E. 1996, AJ, 111, 2415

Strassmeier, K. G. 2009, A\&ARv, 17, 251

Strassmeier, K. G., Carroll, T. A., Weber, M., et al. 2011, A\&A, 535, A98

Trigilio, C., Umana, G., \& Migenes, V. 1993, MNRAS, 260, 903

Umana, G., Trigilio, C., \& Catalano, S. 1998, A\&A, 329, 1010

Umana, G., Trigilio, C., Hjellming, R. M., Catalano, S., \& Rodono, M. 1993, A\&A, 267, 126

White, S. M., Kundu, M. R., Uchida, Y., \& Nitta, N. 1990, in ASP Conf. Ser. 9, Cool Stars, Stellar Systems, and the Sun, ed. G. Wallerstein (San Francisco, CA: ASP), 239 